\title{
LA ADMINISTRACIÓN DE JUSTICIA PENAL EUROPEA Y TRANSNACIONAL COMO DESAFÍO PARA UNA DOGMÁTICA DE DERECHO PENAL MODERNA*
}

Walter Perron ${ }^{* *}$

\section{Resumen}

La discusión jurídico-penal actual se caracteriza por la lenta pero segura pérdida de importancia de los discursos del derecho penal puramente nacionales, frente a la creciente importancia de las elaboraciones del derecho penal europeo e internacional; aquí, justo es decirlo, es donde se plantea la pregunta acerca de qué sucederá en el futuro con la dogmática de derecho penal alemana y su larga tradición, máxime si se tiene en cuenta que en la propia Alemania la importancia de los grandes edificios teóricos ha disminuido. Además, dado que no es posible prever, si y / o cuando se unirán los estados miembros de la Unión Europea para la creación de un "derecho penal europeo" supranacional directamente aplicable, el cual reemplace por lo menos parcialmente al derecho penal nacional, es de prever que esta alzadura teórica continúe, aunque tendrá que adaptarse a los nuevos desarrollos. En cualquier caso, el autor estima que, en el porvenir, de la dogmática de derecho penal habrá de hacerse teoría del derecho penal.

\section{Palabras claves}

Derecho penal, Dogmática, política criminal, derecho penal internacional, derecho europeo, administración de justicia.

\begin{abstract}
The current legal-criminal discussion is characterized by the slow but sure loss of importance of purely national criminal law discourses, in the face of the growing importance of the elaborations of European and international criminal law; here,
\end{abstract}

* Título original: "Europäische und Transnationale Strafrechtspflege als Herausforderung für eine moderne Strafrechtsdogmatik", traducción a cargo de Jhaslen Ricardo Ramírez Lemus (Albert-Ludwigs-Universität Freiburg \& Universidad de Málaga). El presente artículo hace parte del texto de Tiedemann, K., Sieber, U., Satzger, H., Burchard, Ch \& Brodowski, D. (eds.), Die Verfassung moderner Strafrechtspflege. Erinnerung an Joachim Vogel, Baden-Baden 2016, 307-320.

** Estudios de Derecho y Doctor en Derecho de la Universidad Albert-Ludwigs-Universität Freiburg; Profesor de Derecho Penal, Derecho Procesal Penal y Derecho Penal comparado de la Universidad Albert-Ludwigs-Universität Freiburg, Alemania; E-Mail: walter.perron@ jura.uni-freiburg.de 
it is fair to say, is where the question arises about what will happen in the future with the dogmatics of German criminal law and its long tradition, especially taking that in Germany itself the importance of large theoretical buildings has diminished. Furthermore, given that it is not possible to foresee, if and / or when the member states of the European Union will be united for the creation of a directly applicable supranational "European criminal law", which at least partially replaces the national criminal law, it is to foresee that this theoretical elevation continues, although it will have to adapt to new developments. In any case, the author considers that, in the future, criminal law theory will have to be made of criminal law dogmatics.

\section{Keywords}

Criminal law / Dogmatics / criminal policy / international criminal law / European law / administration of justice.

\section{Introducción}

En un artículo publicado en la revista JZ en 2012, Joachim Vogel había profetizado una especie de "schock de Pisa" en el caso de que la ciencia de derecho penal alemana no abandonase su desmesurada autoestima y tomase nota de la realidad internacional (Vogel, 2012, p. 25). Bajo su perspectiva, la influencia del pensamiento jurídico alemán sobre los desarrollos internacionales es escasa y la ciencia de derecho penal alemana solo es tenida en consideración en Estados "que como Italia, España, Grecia o Japón, cuentan con un pasado problemático común con Alemania" (Vogel, 2012, p. 27). Los ejemplos a los que él acudió como prueba de este diagnóstico resultan convincentes; así, en particular, las condenas a Alemania por parte del Tribunal Europeo de Derechos Humanos (TEDH) en tiempos recientes o la inexistente influencia tanto de la tradición como de los conceptos de derecho alemanes en actos, convenciones e instrumentos jurídicos internacionales. No obstante, cabe preguntarse si esta valoración no resulta demasiado pesimista.

\section{Dogmática de derecho penal alemana y sistemas internacionales multinivel}

Para poder comprender el significado que tienen los desarrollos internacionales en la dogmática de derecho penal, primero hay que tener claro qué es ésta en realidad. Al respecto, Hans Joachim Hirsch, en el marco de un coloquio multinacional alemán, italiano, portugués y español llevado a cabo en 1990, afirmó de manera provocadora: "Según estándares científicos no existe una dogmática de derecho penal italiana, portuguesa, española o alemana, sino solo una total o parcialmente correcta, o, por el contrario, una errada" (Hirsch, 1991, p. 27). Hirsch justificó su afirmación aseverando que la dogmática científica investiga: "cuáles son los requisitos que debe presentar un hecho punible y cómo deben estructurarse" (1991, p. 54, nota 96).

Considero que esta postura es errónea. En mi opinión, la dogmática del derecho en general y la dogmática de derecho penal en particular no pueden prescindir del ordenamiento jurídico concreto al cual se relacionan. Al respecto, no se trata únicamente de los requisitos de un hecho punible, sino, sobre todo, de cómo estos 
conocimientos generales repercuten sobre la interpretación de las disposiciones concretas de un ordenamiento jurídico y su aplicación en un caso específico. La tarea de la dogmática es la elaboración de una conexión entre los conocimientos abstractos generales y las leyes aplicables, de cara a la solución concreta de casos jurídicos individuales, para así crear un sistema lo más consistente y libre de contradicciones posible, el cual haga compatibles, en la mayor medida posible, a la seguridad jurídica y a la justicia para cada caso en concreto.

Así lo declaró el escrito "Perspectivas de la ciencia del derecho en Alemania", el cual fue elaborado por el Consejo Científico": "La ciencia del derecho desarrolla la teoría acerca del contenido del derecho vigente (dogmática[s])...La ciencia del derecho alemana se caracteriza, particularmente, por tratar la totalidad del derecho de una manera amplia, conceptual y sistemática" (Wissenschaftrat, 2012, p. 25). Consecuentemente, la dogmática del derecho es relacionada con el derecho positivo y lo desarrolla de manera amplia a través de un trabajo de elaboración conceptual y sistemático. Ésta se sitúa, por un lado, entre un positivismo legal puro, el cual aplica el derecho vigente sin adelantar una discusión crítica; y, por otro lado, dentro de la teoría general del derecho, la cual busca explicar, desprendida de las particularidades de un ordenamiento particular, la totalidad de las formas de manifestación del derecho. Por lo demás, una definición semejante es similar al concepto teológico de la dogmática, el cual, partiendo de principios centrales de la teoría del pensamiento cristiano, se encarga de "la descripción sistemática de la totalidad de las verdades de la revelación" (Ruster, 2005, p. 2).

Esto significa, para nuestro tema, que la dogmática de derecho penal siempre está relacionada a un sistema jurídico positivo, aunque no lo aplica directamente, sino que permite la continuación de su desarrollo y asesora al legislador de manera prospectiva a través del recurso a principios generales y al derecho suprapositi$\mathrm{vo}^{2}$, acompañándolos de perspectivas y razonamientos con talante crítico. Es por ello que la dogmática de derecho penal alemana está relacionada al derecho penal alemán y, ciertamente, desde esta perspectiva, no debería ocuparse del desarrollo internacional.

Naturalmente, una visión así no puede ser defendida hoy en día sin una fuerte limitación. El legislador nacional y los tribunales nacionales han dejado de ser soberanos en la adopción y aplicación del derecho penal y están ligados ahora en gran medida a las disposiciones del derecho internacional. Los acuerdos, decisiones marco y directivas europeas fijan el contenido a muchas, cuando no a la mayoría, de las normas de derecho penal de tiempos recientes y únicamente dejan al legislador la configuración de su modo o forma concretos (Ambos, 2014, 11,15 y ss.; Esser, 2014, 2, 135 y ss.; Hecker, 2012, 11, 1 y ss.; Safferling, 2011, 10, 47 y ss.; Satzger, 2013, 9, 31 y ss.). En la aplicación de dichas leyes, los tribunales también se ven

\footnotetext{
${ }^{1}$ El Consejo Científico (Wissenschaftsrat en alemán) es un organismo encargado de asesorar las políticas científicas en Alemania en temas tales como el desarrollo de universidades, de la ciencia y de la investigación (N. del T.).

${ }^{2}$ La expresión se utiliza en relación con el derecho natural (N. del T.).
} 
frecuentemente obligados a tener en cuenta las disposiciones europeas a través de una interpretación conforme al derecho europeo de cara a la decisión de casos individuales (Hecker, 2012, 10, 1 y ss., con numerosa jurisprudencia del TEDH y los tribunales alemanes; fundamental para Alemania fue la decisión del Bundesgerichtshof BGHSt 37, 333, [336]); y, además, las decisiones del Tribunal Europeo de Derechos Humanos repercuten de manera duradera tanto en la legislación como en la jurisprudencia, incluso si Alemania no tomó parte del procedimiento correspondiente (Ambos, 2014, 10, 8). Finalmente, Alemania ha ratificado muchos acuerdos internacionales fuera del espacio jurídico europeo, lo cuales obligan a los países signatarios a la creación de normas penales con contenidos específicos; así, por ejemplo, en el ámbito del derecho penal relacionado a sustancias estupefacientes, la Convención de las Naciones Unidas contra el tráfico ilícito de estupefacientes y sustancias sicotrópicas de 1988 (BGB1.1993 II S. 1136).

Dicho en otras palabras: el legislador alemán ha depositado parte de su soberanía en la Unión Europea y en Organizaciones Internacionales u Órganos de derecho internacional que surgen de sus tratados. Por ende, la dogmática de derecho penal alemana debe ocuparse del trabajo de elaboración de su propio derecho bajo esta influencia internacional. De hecho, esta discusión tiene cada vez más lugar tanto en los comentarios como en los artículos y en la literatura de los manuales alemanes, así como lo demuestra la discusión actual respecto de la delimitación del §263 del StGB (Código Penal alemán) a la luz del derecho de protección al consumidor europeo (al respecto, en tiempos recientes: Klesczweski, 2014, p. 1085; Tiedemann, 2014, pp. 1107, 1111; también, BGH NJW 2014, pp. 2595 y ss.). De todas formas, los correspondientes comentarios se ocupan casi de manera exclusiva de la pregunta acerca de las consecuencias que, de cara a la aplicación del derecho, han de extraerse a partir de las disposiciones internacionales; mientras que, en el mejor de los casos, una discusión respecto de la exactitud e idoneidad de estas disposiciones solo tiene lugar en aquellas contribuciones que se orientan al campo de la política criminal. Así, primero, la iniciativa para un "Corpus Juris" (Delmas-Marty \& Vervaele, 2000), así como, recientemente y en particular, el grupo formado por Petter Asp et al. (2009) con el texto "A Manifesto on European Criminal Procedure Law" y "The European Public Prosecutor's Office" (2015).

\section{Dogmática de derecho penal europea e internacional}

Es solo lentamente y con dificultades como cada vez tiene más aceptación la idea de que los diferentes "niveles" internacionales que influyen al derecho penal demuestran rasgos característicos propios de un "ordenamiento jurídico" y por ello requieren una "dogmática" propia. Para la ilustración de este desarrollo quisiera mencionar tres ámbitos: el de la Unión Europea, el de la Convención Europea de los Derechos Humanos y el de la Corte Penal Internacional.

Sobre la posición del derecho penal en la Unión Europea se ha escrito y discutido bastante en Alemania (Ambos, 2014, 9, 1; Hecker, 2012, desde 1, 43, a 4, 84; Satzger, 2013, 9, 116; Safferling, 2011, 10, 110). Normalmente, los autores se ocupan de temas como la creación o interpretación de disposiciones jurídicas alemanas conforme al 
derecho europeo, o del régimen de competencias de la UE. Estas perspectivas son comprensibles, dado que, en el pasado reciente, el principal instrumento jurídico de la UE en el plano del derecho penal fue la decisión marco creada a través del Tratado de Ámsterdam de 1999, la cual fue a su vez sustituida vía directiva a partir del tratado de Lisboa. Ninguno de los dos, salvo excepciones, cobran efecto de manera directa, sino que requieren de su adopción por parte del legislador nacional. Además, las discusiones en materia de política criminal respecto del contenido de las decisiones marco fueron escasamente llevadas a cabo, debido al principio de unanimidad y a la dinámica específica del proceso para lograr acuerdos al interior del Consejo de la Unión Europea, donde los gobiernos nacionales no destaparon sus verdaderas posturas en el período previo a dichas decisiones. Naturalmente, esto ha cambiado fundamentalmente a partir del tratado de Lisboa: La Comisión Europea tiene ahora el derecho de iniciativa y a menudo presenta proyectos que son discutidos de manera pública (Comisión Europea, 2015), las votaciones en el Parlamento y en el Consejo son decididas por medio de mayorías -simples o cualificadas- (art. 16 IV EUV, art. 231, 238, 294 AEUV), de manera que las tácticas de secretismo son menos necesarias; y sobre todo, ahora es posible llevar a cabo discusiones públicas de forma previa a la toma de decisiones en el Parlamento Europeo, discusiones que, además, implican también a especialistas en el área; un ejemplo de ello es el procedimiento del Parlamento llevado para la Orden Europea de Investigación en materia penal (Parlamento Europeo, 2014; sobre ello, Böse, 2014, p. 152, Schuster, 2015, p. 393).

A esto ha de añadirse que, a partir del Tratado de Ámsterdam, el Tribunal de Justicia de la Unión Europea tiene competencia para decidir sobre el contenido e interpretación de decisiones marco y directivas penales (art. 19 Abs. 3 EUV, art. 267 AEUV). Dado que estos actos jurídicos utilizan frecuentemente conceptos penales clave como: "instigación", "autoría" o "tentativa" (cfr. por ejemplo, el art. 4 de la Decisión Marco del 13.6.2002 sobre la lucha contra el terrorismo, AB1.L 164/3), el acceso con que cuenta el TJUE respecto de los fundamentos de la dogmática de derecho penal está abierto. Hasta ahora el tribunal de Luxemburgo ha hecho uso de esta posibilidad en menor medida, pero esto puede cambiar en el futuro rápidamente.

Finalmente, cada vez se encuentran más signos para la apertura de un discurso científico europeo respecto del derecho penal europeo. Al principio solían encontrarse en primer plano iniciativas individuales tales como el proyecto "Corpus-juris" o recientemente el "Manifiesto europeo" (la prueba de ello, el derecho contra los estupefacientes: la Convención de las Naciones Unidas contra el tráfico ilícito de estupefacientes y sustancias sicotrópicas de 1988). Hoy día, por su parte, la creación de revistas europeas específicas conllevó a un creciente intercambio internacional (véanse las publicaciones citadas infra). Esto se hace palpable también, por ejemplo, en las solicitudes de subvención extranjeras dirigidas a la Fundación Alexander von Humboldt, sobre todo en el campo del derecho procesal penal, donde se presta cada vez más atención al derecho penal europeo y cuyos solicitantes presentan frecuentemente publicaciones en lengua inglesa. 
Si se compendian estos desarrollos, no puede pasarse por alto el hecho de que se está desarrollando una especie de ordenamiento jurídico-penal en el plano de la Unión Europea. Aunque los ámbitos de regulación son aún muy fragmentarios y los objetos y alcances de estas discusiones son limitadas, los planos relativos a la legislación, la jurisprudencia y a la discusión científica se establecen y crecen paulatinamente de manera conjunta.

Aún más claro es el desarrollo en el campo de la Convención Europea de los Derechos Humanos. Sobre todo, después de la gran reforma a los tribunales de 1998, llevada a cabo a través del 11. Protocolo adicional a la CEDH del 11.5.1994, en vigor desde el 1.11.1998, los magistrados de Estrasburgo han aumentado considerablemente su confianza en sí mismos, al punto que ya no evitan conflictos con instancias nacionales altamente respetadas, como por ejemplo el tribunal federal constitucional alemán, y se están imponiendo paulatina y decididamente frente a todos los Estados miembros. Ello a pesar de que el ámbito de regulación de esta convención está fuertemente delimitado, pues solo contiene unas pocas garantías mínimas. Por otro lado, este instrumento cuenta con la figura de la demanda individual, la cual brinda a cada persona el derecho de demandar a un Estado miembro frente al TEDH (art. $34 \mathrm{CEDH}$ ), situación que conllevó a una oleada de procesos que en gran número desembocaron en importantes decisiones y que, en particular, influyen en el derecho procesal penal de manera duradera (cfr., por ejemplo, la compilación de sentencias en el idioma alemán en la página web del tribunal). Es así como desde hace algún tiempo entran en reiteradas discusiones las decisiones del tribunal federal frente a la jurisprudencia del tribunal de Estrasburgo (por ejemplo, BGHSt (GS) 52, 124, 131).

En el pasado, la discusión científica acerca de la CEDH y de la jurisprudencia del TEDH fue llevada a cabo principalmente por los representantes del derecho internacional público (Dörr, Grote \& Marauhn, 2013; Frowein \& Peukert, 2009; Grabenwarter \& Pabel, 2015), mientras que, por otra parte, los penalistas alemanes se limitaron a la perspectiva nacional y, por ejemplo, se preguntaban si el art. 2 de la CEDH conllevaba a una limitación del derecho a la legítima defensa garantizado por el §32 del StGB (comunes, en este sentido, son los comentarios de Lenckner, 1997, 32, 62; una opinión diferente se encuentra en mis comentarios, 2014). En cambio, hoy día existe ya una discusión internacional relacionada al derecho penal de cara a las decisiones centrales del TEDH y su repercusión en los sistemas de derecho nacionales (cfr., por ejemplo, los aportes en la revista Human Rights Law Review editada por Oxford; otras pruebas sobre aportes en lengua inglesa en $\mathrm{Am}$ bos, 2014, 10,1).

El estatuto de Roma de la Corte Penal Internacional representa un ordenamiento jurídico completo en sí mismo. Este estatuto no solamente regula la organización de la Corte, sino que incluye todas las disposiciones de derecho penal sustancial y procesal que esta ha de aplicar (Rome Statute, 2002). Se trata por ello de un área que exige el establecimiento y diferenciación de una dogmática de derecho penal internacional. $\mathrm{Al}$ respecto se encuentran numerosas publicaciones científicas, de 
momento casi todas en el idioma inglés, que se ocupan de manera detallada de las decisiones de la CPI (cfr. tan solo el índice de literatura en Satzger, 2013, §§ 12-17, que en muchos casos también tienen que ver con autores alemanes que hacen aportaciones en el idioma inglés). Incluso tratados y manuales de relevancia central y monografías aparecen publicadas en inglés (Ambos, 2013-2014; Werle \& Jeßberger, 2014). La única razón por la cual su desarrollo no se encuentra aún muy avanzado, se debe a la todavía joven historia de esta Corte y al reducido número tanto de procesos como de decisiones emitidas hasta ahora.

En este contexto cabe preguntarse si no existe ya una dogmática de derecho penal europea o internacional y, de haberla, qué aspecto tiene. A este respecto ha de dejarse claro que en el idioma inglés el concepto "dogmática", esto es, "dogmatics" no es usado fuera del ámbito de la teología. Es por ello que el intento de desarrollar una dogmática internacional de derecho penal ${ }^{3}$ de corte universal liderado desde el lado alemán, ha de ser ya vista, desde el punto de vista del idioma, como infructuosa. Por otra parte, en todos los planos nombrados existe una incuestionable necesidad de investigar y concretar el contenido y significado de las disposiciones jurídicas internacionales a través de la interpretación acerca de qué deben hacer estos tribunales. A través de la discusión crítica tanto de estas disposiciones jurídicas como de su aplicación judicial, llevada a cabo en el marco de foros científicos y medios especializados, es como se origina de manera más o menos forzosa un discurso transnacional, el cual busca comprender las disposiciones y decisiones individuales en su contexto; $y$, además, formula sistematizaciones, si bien es claro que en muchos lugares se adelantan planteamientos claramente más pragmáticos que los de la ciencia de derecho penal alemana, más orientada a los principios.

Ciertamente, o bien existen ya varias "dogmáticas internacionales de derecho penal" o bien se encuentran en su devenir. Sin embargo, estas difieren significativamente de nuestra tradición alemana. Esto comienza ya con el idioma: también en el plano internacional, el inglés se ha introducido en la ciencia del derecho. Este permite, por un lado, el uso de formas de expresión más directas y más sencillas que las complicadas construcciones de oraciones que caracterizan al idioma alemán. Por otro lado, no obstante, se encuentra muy limitado al mundo conceptual del Common Law. Es por ello que deben ser encontrados nuevos conceptos y definiciones para evitar malentendidos en el discurso jurídico internacional; así, por ejemplo, el art. 30 del Estatuto de Roma habla sobre un "mental element" y no de una "mens rea", el art. 31, por su parte, habla de "grounds for excluding criminal responsability" y no de "defences". A pesar de estas dificultades rige lo siguiente: quien busca ser escuchado en la discusión internacional, debe publicar en inglés, pues

\footnotetext{
${ }^{3}$ La frase utilizada por el autor es: Internationale Strafrechtsdogmatik, la cual pudiese también ser traducida como "dogmática de derecho penal internacional". Sin embargo, con el ánimo de evitar limitar su sentido al "derecho penal internacional", esto es, al relacionado a la CPI o a los tribunales ad hoc de La ONU; y teniendo en cuenta que el autor se refiere a una dogmática surgida desde el plano internacional para el derecho penal, se ha preferido la expresión "dogmática internacional de derecho penal” (N. del T.).
} 
las aportaciones en otros idiomas serán apenas percibidas, pese a ser publicadas en revistas de amplia tradición.

El lugar de publicación tampoco puede ser elegido según la práctica nacional, sino que ha de dirigirse a las revistas internacionales pertinentes y admitir sus condiciones, esto es y en específico, la "peer review". Junto a las tradicionales revistas de habla inglesa internacionalmente orientadas, como por ejemplo la revista estadounidense Harvard International Law Journal, o la británica Human Rights Law Review, recientemente han sido creadas numerosas revistas con un enfoque transnacional europeo, con énfasis en derecho penal, a saber: Journal of Internacional Criminal Justice, New Journal of European Criminal Law, European Journal of Crime, Criminal Law and Justice, European Criminal Law Review, The European Criminal Law Association's Forum o la International Criminal Law Review. Es en estas revistas donde son llevadas a cabo las principales discusiones, mientras que las revistas de habla alemana, como por ejemplo ZIS-online, donde solo de manera ocasional se publican aportaciones elaboradas en inglés, tienen consecuentemente menos influencia.

Finalmente, ya no es posible el apoyarse en grandes edificios o relaciones teóricas que atraviesen las naciones, de la forma en que por ejemplo lo hace la teoría del delito alemana con su separación entre injusto y culpabilidad, pues ese tipo de edificios teóricos cuentan normalmente con un fuerte carácter nacional y además son desconocidos por muchos lectores cuya procedencia es distinta. Me refiero, en específico, por quienes provienen del mundo angloparlante. El estilo argumentativo en el discurso internacional es más bien pragmático y sobre todo orientado al argumento concreto, mientras que la referencia a autoridades en la materia solo tiene sentido si dicho autor se ha logrado consolidar como tal en el nivel internacional.

La mejor forma de apreciar el estilo del discurso internacional del derecho penal puede ser constatada en los equipos de colaboradores científicos de los tribunales europeos e internacionales. La conformación de estos equipos es multinacional y por lo general se compone de jóvenes juristas, cuya formación universitaria nacional no culminó mucho tiempo atrás y que, además, no han desarrollado una lealtad lo suficientemente fuerte frente a su propio sistema. El foco de su actividad se centra en las tareas concretas que afrontan, esto es, a la clasificación de los casos que arriben de acuerdo a la jurisprudencia existente y a la preparación de propuestas de solución para nuevas preguntas que aún no hayan sido resueltas. Para llevar a cabo esta tarea, tanto el TJUE como el TEDH y la CPI han desarrollado un enfoque sistemático, aunque éste no es determinado por el discurso científico sino por las decisiones del tribunal correspondiente. Por los pasillos de estos tribunales tienen lugar vivas discusiones, en las cuales es poco probable el constatar quién proviene de uno $\mathrm{u}$ otro sistema nacional. Quien desee encontrar aquí un auditorio o ganar influencia, ha de adaptarse a este tipo de cultura de discusión y, en particular, ha de brindar argumentos prácticos al tribunal correspondiente, de manera que estos puedan ser tomados directamente por los Magistrados en sus decisiones. 


\section{El futuro de la dogmática de derecho penal alemana}

En el contexto de esta lenta pero segura pérdida de importancia de los discursos de derecho penal puramente nacionales frente a la creciente importancia del discurso del derecho penal europeo e internacional, es donde se plantea la pregunta acerca de qué sucederá con la dogmática de derecho penal alemana y su larga tradición. En primer lugar, ha de constatarse cómo parece ser que incluso en Alemania la importancia de los grandes edificios teóricos ha disminuido. Pese a que, recientemente, Michael Pawlik ha presentado con su "injusto del ciudadano", siguiendo la tradición de su maestro Günther Jakobs, un escrito esencial para la construcción de la imputación penal (2012), el día a día de un penalista alemán está principalmente caracterizado, por el contrario, por la realización de comentarios a leyes y a sentencias, todos los cuales, tal cual ocurre en el área del derecho penal económico, tratan en vano de seguir la pista a los vertiginosos desarrollos legislativos y jurisprudenciales. Incluso los manuales de las últimas décadas están orientados casi exclusivamente a la mediación didáctica y poco ya a la fundamentación teórica (los últimos manuales cuya pretensión se orienta a los fundamentos son los de Jakobs, 1993; Köhler, 1997; Roxin, I, 2006; Roxin, 2, 2003).

Pese a este clima más bien orientado hacia la práctica, la dogmática de derecho penal alemana todavía puede continuar funcionando en el formato actual por mucho más tiempo. Dado que no es posible prever, si y/o cuando se unirán los estados miembros de la Unión Europea para la creación de un "derecho penal europeo" supranacional directamente aplicable, el cual reemplace por lo menos parcialmente al derecho penal nacional, la necesidad de contar con comentarios y sistematizaciones nacionales permanecerá inalterada desde la perspectiva de los usuarios del derecho.

Por otra parte, sobre todo los jóvenes científicos no podrán permitirse el lujo de ignorar completamente el plano europeo. Del mismo modo como en el derecho público hoy día el derecho estatal no puede ser enseñado e investigado sin referencia al derecho europeo, así también será el caso para el derecho penal, donde el recurso directo a las disposiciones y normas de derecho europeo pertenecerán al día a día de su dogmática. A la par aumentará marcadamente el número de especialistas que se dedicarán exclusivamente a las diferentes dimensiones internacionales del derecho penal y que publicarán cada vez más en el plano internacional.

Sin embargo, en este clima cambiante, la pregunta verdaderamente interesante gira en torno al "si" y al "cómo" persistirán aquellas tradiciones que han afamado a la ciencia de derecho penal alemana en muchos lugares del mundo. Se trata, por un lado, de la dogmática de derecho penal orientada a los fundamentos, en la que desde la segunda guerra mundial han existido nombres tales como Hans Welzel, Hans-Heinrich Jescheck, Claus Roxin, Günther Jakobs o Ernst Amadeus Wolff, y, por el otro, del impulso político-criminal de Franz v. Liszt y el "Proyecto alternativo" -así denominado en su época por su círculo de trabajo- el cual fue y es personificado en Jürgen Baumann, todos los cuales contribuyeron activamente a dar forma al ulterior desarrollo del derecho penal (Arbeitskreis Alternativ-Entwurf). Ambas 
tradiciones son aún hoy continuadas en el plano nacional, sin embargo, estas ya no podrán desarrollar más su mismo magnetismo sin relación al plano internacional.

Y es que así han cambiado los intereses, sobre todo de los científicos extranjeros, quienes se hayan centrados con cada vez más fuerza en el plano internacional y europeo, siendo que allí no encuentra auditorio una dogmática planteada en el idioma y con relación al derecho alemán. A su vez, la configuración político-jurídica del derecho penal se lleva a cabo cada vez más desde un escenario político internacional, al cual los círculos de trabajo alemanes, en el mejor de los casos, apenas si tienen acceso indirecto a través puntos de enlace nacionales, mientras que los grupos internacionales, cuya composición científica es plurinacional, mantienen de manera mucho más sencilla un acceso directo a las instituciones europeas (así, por ejemplo, los citados grupos liderados por Delmas-Marty \& Vervele, 2000 y Asp, 2009 y 2015).

Si se quiere que la larga tradición de la ciencia de derecho penal alemana prosiga de manera exitosa en el futuro, entonces esta ha de adaptarse a las circunstancias cambiantes. La perspectiva orientada a los fundamentos debe despegarse del derecho positivo alemán y tomar en consideración la diversidad de ordenamientos e instituciones posibles con ayuda del derecho comparado (al respecto, en particular el aporte de Tatjana Hörnle, 2016, p. 289 y ss.). Por ejemplo, la discusión alemana respecto de la delimitación entre el dolo eventual y la culpa consciente se relativiza claramente, si se la confronta de manera imparcial con las categorías anglosajonas de Intention, Recklesness y Negligence, y se procede a indagar a partir de qué datos psicológicos debe establecerse efectivamente una mayor punibilidad para el delito doloso (cfr. al respecto, Perron, 1998, p. 145 y ss.). Dicho de una manera más sencilla: de la dogmática de derecho penal habrá de hacerse teoría del derecho penal. Si esta teoría es directamente planteada en el idioma inglés o si primero es publicada en alemán y luego traducida al inglés (y también a otros idiomas), considero que es poco relevante, siempre y cuando dichos trabajos sean elaborados de una manera accesible para un público más amplio, el cual no comprenda el idioma alemán; aún hoy, la gran relevancia de la dogmática de derecho penal alemana en España, Latinoamérica y el Asia oriental se basa sobre todo en la traducción de obras centrales al español, japonés, coreano o al chino-mandarín.

Respecto de la influencia político-criminal, ha de tenerse en cuenta que en el escenario internacional solamente serán efectivos los gremios y círculos de trabajo internacionales cuyas propuestas en el plano político sean redactadas en inglés. Dichos círculos de trabajo existen ya y tanto compañeras como compañeros alemanes participan en ellos de manera decisiva. Lo mismo vale para el compromiso alemán con las revistas internacionales recientemente creadas. Si se observan con detalle estos desarrollos, bien puede asegurarse que la transformación por mí reclamada ya está teniendo lugar y que, por lo menos bajo las apariencias, es operada de forma sostenible. Por lo tanto, no tengo ninguna duda de que la ciencia de derecho penal alemana estará a la altura de los desafíos de la administración de justicia penal europea y transnacional. 


\section{Referencias}

Ambos, K. (2013-2014). Treatise on International Criminal Law, 3 vols. Oxford: Oxford University Press.

Ambos, K. (2018). Internationales Strafrecht, 5a. ed., München: C. H. Beck.

Arbeitskreis Alternativ-Entwurf. Recuperado de https:/ /blogs.hhu.de/alternativentwurf/

Asp, P. et al. (2009). European Criminal Policy Initiative: A Manifesto on European Criminal Procedure Law. Recuperado de http:/ / www.zis-online.com/dat/artikel/2009_12_383.pdf

Asp, P. (2015). The European Public Prosecutor's Office: legal and criminal policy perspectives / European Criminal Policy Initiative. Stockholm: Stiftelsen Skrifter utgivna av Juridiska fakulteten vid Stockholms universitet.

Böse, M. (2014). Die Europäische Ermittlungsanordnung - Beweistransfer nach neuen Regeln? En Zeitschrift für Internationale Strafrechtsdogmatik, N. ${ }^{\circ}$ 4, 152-164. Recuperado de http: / / www.zis-online.com/dat/artikel/2014_4_808.pdf

Bundesgerichtshof (2014, marzo cinco). 2- StR 616/12 “Abo-Falle" im Internet als Versuchter Betrug - Routenplaner. En Neue Juristische Wochenschrift, N. ${ }^{\circ}$ 35, 2595-2599.

Comisión Europea (2015, septiembre 17). Compilación de la base de datos Eurocrim de Tübingen. Recuperado de http:/ / www.europarl.europa.eu/sides/getDoc. do type $=$ CRE\&reference $=20140226 \&$ secondRef $=I T E M-017 \&$ language $=$ DE\&rin $\mathrm{g}=\mathrm{A} 7-2013-0477$

Delmas-Marty, M. \& Vervaele, J. A. E. (eds.) (2000-2001). The Implementation of the Corpus Juris in the Member States, 4 vols. Antwerpen: Intersentia.

Dörr, O, Grote, R. \& Marauhn, Th. (2013). EGMR/GG: Konkordanzkommentar zum europäsichen und deutschen Grundrechtsschutz, 2a. ed. Tübingen: Mohr Siebeck.

Esser, R. (2014). Europäisches und Internationales Strafrecht. München: C. H. Beck.

Eser, A. \& Perron, W. (eds.) (1991). Rechtfertigung und Entschuldigung III, Deutschitalienisch-portugiesisch-spanisches Strafrechtskolloquium 1990. Freiburg: Max Planck Institut für ausländisches und Internationales Straftrecht.

Frowein, J. \& Peukert, W. (2009). Europäische Menschenrechtskonvention (3a. ed.). Berlin: Engel Verlag.

Grabenwarter, Ch. \& Pabel, K. (2015). Europäische Menschenrechtskonvention (6a. ed.). München: C. H. Beck. 
Hörnle, T. (2016). Plädoyer für eine transnationale Strafrechtswissenschaft. En Tiedemann, K., Sieber, U., Satzger, H., Burchard, Ch \& Brodowski, D. (Edits.), Die Verfassung moderner Strafrechtspflege. Erinnerung an Joachim Vogel (pp. 289306), Baden-Baden: Nomos.

Hecker, B. (2012): Europäisches Strafrecht (4a. ed.). Berlin-Heidelberg: Springer Verlag.

Hirsch, H. J. (1991). Die Stellung von Rechtfertigung und Entschuldigung im Verbrechenssystem aus deutscher Sicht. En Eser, A./Perron, W. (Edits.), Rechtfertigung und Entschuldigung III (pp. 27-54), Freiburg: Max-Planck Institut für ausländisches und internationales Strafrecht.

Jakobs, G. (1993). Strafrecht Allgemeiner Teil (2a. ed.). Berlin-New York: Walter de Gruyter.

Klesczweski, D. (2014). Zur europarechtlichen Restriktion des Betrugstatbestandes. En Hefendehl, R., Hörnle, T. \& Greco, L., Festschrift für Bernd Schünemann zum 70. Geburtstag am 1. November 2014: Streitbare Strafrechtswissenschaft (pp. 10851098). Berlin: De Gruyter.

Köhler, Michael (1997). Strafrecht. Allgemeiner Teil, Berlin-Heidelberg-New York: Springer Verlag.

Parlamento Europeo (2014). Directiva 2014 / 41/CE del Parlamento Europeo y del Consejo, de 3 de abril de 2014, relativa a la orden europea de investigación en materia penal. Recuperado de https://eur-lex.europa.eu/legal-content/ES/ TXT / ?uri=celex\%3A32014L0041

Parlamento Europeo (2014, febrero 26). Debate sobre Orden Europea de Investigación en materia penal. Recuperado de http:/ / www.europarl.europa. eu $/$ sides $/$ getDoc.do?type $=$ CRE\&reference $=20140226 \&$ second Ref=ITEM017\&language $=\mathrm{DE} \&$ ring $=\mathrm{A} 7-2013-0477$

Pawlik, M. (2012). Das Unrecht des Bürgers, Grundlinien der Allgemeinen Verbrechenslehre. Tübingen: Mohr Siebeck.

Perron, W. (1998). Vorüberlegungen zur einer rechtsvergleichenden Untersuchung der Abgrenzung von Vorsatz und Fahrlässigkeit. En: A. Eser et al. (Eds.), Festschrift für Haruo Nishihara (pp. 145-156). Baden-Baden: Nomos Verlag.

Perron, W. (2016). Europäische und Transnationale Strafrechtspflege als Herausforderung für eine moderne Strafrechtsdogmatik. En Tiedemann, K., Sieber, U., Satzger, H., Burchard, Ch \& Brodowski, D. (Edits.), Die Verfassung moderner Strafrechtspflege. Erinnerung an Joachim Vogel (pp. 307-319), BadenBaden: Nomos.

Rome Statute of the International Criminal Court (2002, julio 1). Recuperado de http:/ / www.icc-cpi.int/NR/ rdonlyres/ADD16852-AEE9-4757-ABE79CDC7CF02886/283503/RomeStatutEng1.pdf 
Roxin, C. (2006). Strafrecht, Allgemeiner Teil, t. I, Grundlagen, Der Aufbau der Verbrechenslehre (4a. ed.). München: Verlag C. H. Beck.

Roxin, C. (2003). Strafrecht, Allgemeiner Teil, t. II, Besondere Erscheinungsformen der Straftat, $1^{\text {a }}$ ed. München: Verlag C. H. Beck.

Ruster, Th. (2005). Basiswissen Dogmatik. Dortmund, 2005, 1-45. Recuperado de http:/ / www.fk14.tu-dortmund.de/medien/uploads/Basiswissen_Skript.pdf

Safferling, Ch. (2011). Internationales Strafrecht. Berlin-Heidelberg: Springer Verlag.

Satzger, H. (2013). Europäisches und Internationales Strafrecht (6a. ed.). Baden-Baden: Nomos.

Schuster, F. P. (2015). Die Europäische Ermittlungsanordnung - Möglichkeiten einer gesetzlichen Realisierung, Strafverteidiger N. ${ }^{\circ}$ 6, 393-399.

Schönke/Schröder (1997), Strafgesetzbuch (25a. ed.). München: C. H. Beck.

Schönke/Schröder (2014), Strafgesetzbuch (29a. ed.). München: C. H. Beck.

Tiedemann, K. (2014). Kein Liebesverbot für Brüssel und Straßburg-oder Gedanken zur europarechtsfreundlichen Auslegung im Strafrecht. En Hefendehl, R., Hörnle, T. \& Greco, L., Festschrift für Bernd Schünemann zum 70. Geburtstag am 1. November 2014: Streitbare Strafrechtswissenschaft (pp. 1107-1118). Berlin: De Gruyter.

Tribunal Europeo de Derechos Humanos (2018). Sentencias en idioma alemán. Recuperado de http://www.coe.int/T/D/Menschenrechtsgerichtshof/ Dokumente_auf_Deutsch/Volltext/Urteile/

Vogel, J. (2012). Strafrecht und Strafrechtswissenschaft im internationalen und europäischen Rechtsraum, Juristenzeitung, 25-31.

Werle, G. \& Jeßberger, F. (2014). Principles of International Criminal Law (3a. ed.). Oxford: Oxford University Press.

Wissenschaftsrat (2012, noviembre 9). Perspektiven der Rechtswissenschaft in Deutschland. Situation, Analysen, Empfehlungen. Hamburg: Edición 2558-12. Recuperado de https://www.wissenschaftsrat.de/download/archiv/2558-12. pdf 